\title{
Data for deposition:
}

Table : $d(\mathrm{Si}-\mathrm{Si}), \mathrm{d}(\mathrm{Si}-\mathrm{O})$ and $\mathrm{d}(\mathrm{O}-\mathrm{O})$ of RUB-41 based on the Durbin-Watson corrected intensity data.

\begin{tabular}{|c|c|c|c|c|c|}
\hline (Si1 & )$-(\operatorname{Si1}$ & ): & 3.1855 & ( & 43) \\
\hline (Si1 & $-(\operatorname{Si2}$ & ): & 3.1450 & ( & 42) \\
\hline (Si1 & )$-(\operatorname{Si} 3$ & ): & 3.1068 & ( & 43) \\
\hline (Si1 & )$-(\operatorname{Si} 4$ & ): & 2.9635 & ( & 42) \\
\hline (Si1 & )$-(01$ & ): & 1.5765 & ( & 68) \\
\hline (Si1 & )$-(02$ & ): & 1.5927 & ( & 31) \\
\hline (Si1 & )$-(03$ & ): & 1.6125 & ( & $80)$ \\
\hline (Si1 & )$-(04$ & ): & 1.6053 & ( & 50) \\
\hline (Si2 & )$-(\operatorname{Si} 1$ & ): & 3.1450 & ( & 42) \\
\hline (Si2 & )$-(\operatorname{Si} 3$ & ): & 3.0896 & ( & 46) \\
\hline (Si2 & )$-(\operatorname{Si} 4$ & ): & 2.9605 & ( & 53) \\
\hline (Si2 & )$-(\operatorname{Si} 4$ & ): & 3. 1411 & ( & 45) \\
\hline (Si2 & )$-(01$ & ): & 1.5774 & ( & 73) \\
\hline (Si2 & )$-(05$ & ): & 1.5866 & ( & 65) \\
\hline (Si2 & )$-(06$ & ): & 1.5942 & ( & 64) \\
\hline (Si2 & )$-(07$ & ): & 1.5891 & ( & 64) \\
\hline (Si3 & )$-(\operatorname{Si1}$ & ): & 3.1068 & ( & 43) \\
\hline ( $\mathrm{Si} 3$ & )$-(\operatorname{Si2}$ & ): & 3.0896 & ( & 46) \\
\hline (Si3 & )$-(\mathrm{Si} 3$ & ): & 3.0792 & ( & 48) \\
\hline (Si3 & )$-(\operatorname{Si} 5$ & ): & 3.1097 & ( & 40) \\
\hline (Si3 & )$-(04$ & ): & 1.6089 & ( & 57) \\
\hline (Si3 & )$-(07$ & ): & 1.5997 & ( & 64) \\
\hline (Si3 & )$-(08$ & ): & 1.5916 & ( & 83) \\
\hline (Si3 & )$-(09$ & ): & 1.6085 & ( & 41) \\
\hline ( $\mathrm{Si} 4$ & )$-(\operatorname{Si} 1$ & ): & 2.9635 & ( & 42) \\
\hline ( $\mathrm{Si} 4$ & )$-(\operatorname{Si2}$ & ): & 2.9605 & ( & 53) \\
\hline ( $\mathrm{Si} 4$ & )$-(\operatorname{Si} 2$ & ): & 3.1411 & ( & 45) \\
\hline ( $\mathrm{Si} 4$ & )$-(\operatorname{Si} 5$ & ): & 3. 1051 & ( & 37) \\
\hline ( $\mathrm{Si} 4$ & )$-(03$ & ): & 1.5941 & ( & 68) \\
\hline ( $\mathrm{Si} 4$ & )$-(05$ & ): & 1.5838 & ( & 66) \\
\hline (Si4 & )$-(06$ & ): & 1.5941 & ( & 63) \\
\hline (Si4 & )$-(010$ & ): & 1.5805 & ( & 54) \\
\hline (Si5 & )$-(\operatorname{Si} 3$ & ): & 3.1097 & ( & 40) \\
\hline (Si5 & )$-(\mathrm{Si} 3$ & ): & 3.1097 & ( & 40) \\
\hline (Si5 & )$-(\operatorname{Si} 4$ & ): & 3. 1051 & ( & 37) \\
\hline (Si5 & )$-(\operatorname{Si} 4$ & ): & 3.1051 & ( & 37) \\
\hline (Si5 & )$-(08$ & ): & 1.6046 & ( & 74) \\
\hline (Si5 & )$-(08$ & ): & 1.6046 & ( & 74) \\
\hline (Si5 & )$-(010$ & ): & 1.5930 & ( & 56) \\
\hline (Si5 & )$-(010$ & ): & 1.5930 & ( & 56) \\
\hline (01 & )$-(02$ & ): & 2.5896 & ( & 62) \\
\hline (01 & )$-(03$ & ): & 2.6018 & ( & 102) \\
\hline (01 & )$-(04$ & ): & 2.6013 & ( & 87) \\
\hline (01 & )$-(05$ & ): & 2.5910 & ( & 91) \\
\hline (01 & )$-(06$ & ): & 2.5869 & ( & 84) \\
\hline (01 & )$-(07$ & ): & 2.5828 & ( & $85)$ \\
\hline (02 & )$-(01$ & ): & 2.5896 & ( & 62) \\
\hline (02 & )$-(01$ & ): & 2.5896 & ( & 62) \\
\hline (O2 & )$-(03$ & ): & 2.5797 & ( & 66) \\
\hline (O2 & )$-(03$ & ): & 2.5797 & ( & 66) \\
\hline (O2 & )$-(04$ & ): & 2.6425 & ( & 50) \\
\hline (O2 & )$-(04$ & ): & 2.6425 & ( & 50) \\
\hline (03 & )$-(01$ & ): & 2.6018 & & $102)$ \\
\hline
\end{tabular}




\begin{tabular}{|c|c|c|c|c|c|}
\hline (03 & )$-(02$ & ): & 2.5797 & ( & 66) \\
\hline (03 & )$-(04$ & ): & 2.6289 & ( & $86)$ \\
\hline (O3 & )$-(05$ & ): & 2.5851 & ( & 73) \\
\hline (03 & )$-(06$ & ): & 2.5897 & ( & 94) \\
\hline (03 & )$-(010$ & ): & 2.6196 & & 78) \\
\hline (04 & )$-(01$ & ): & 2.6013 & ( & 87) \\
\hline (O4 & )$-(02$ & ): & 2.6425 & ( & 50) \\
\hline (O4 & )$-(03$ & ): & 2.6289 & ( & 86) \\
\hline (O4 & )$-(07$ & ): & 2.6236 & ( & 77) \\
\hline (O4 & )$-(08$ & ): & 2.6285 & ( & 87) \\
\hline (O4 & )$-(09$ & ): & 2.6565 & ( & 54) \\
\hline (05 & )$-(01$ & ): & 2.5910 & ( & 91) \\
\hline (05 & )$-(03$ & ): & 2.5851 & ( & 73) \\
\hline (05 & )$-(05$ & ): & 3.0837 & ( & 117) \\
\hline (05 & )$-(06$ & ): & 2.5676 & ( & 87) \\
\hline (05 & )$-(06$ & ): & 2.6532 & ( & 90) \\
\hline (05 & )$-(07$ & ): & 2.5975 & ( & 80) \\
\hline (05 & )$-(010$ & ): & 2.5229 & ( & 69) \\
\hline (06 & )$-(01$ & ): & 2.5869 & ( & 84) \\
\hline (06 & )$-(03$ & ): & 2.5897 & ( & 94) \\
\hline (06 & )$-(05$ & ): & 2.5676 & ( & 87) \\
\hline (06 & )$-(05$ & ): & 2.6532 & ( & 90) \\
\hline (06 & )$-(07$ & ): & 2.6213 & ( & 89) \\
\hline (06 & )$-(010$ & ): & 2.5874 & ( & 87) \\
\hline (07 & )$-(01$ & ): & 2.5828 & ( & $85)$ \\
\hline (O) & )$-(04$ & ): & 2.6236 & ( & 77) \\
\hline (07 & )$-(05$ & ): & 2.5975 & ( & 80) \\
\hline (07 & )$-(06$ & ): & 2.6213 & ( & 89) \\
\hline (O7 & )$-(08$ & ): & 2.5920 & ( & 102) \\
\hline (O7 & )$-(09$ & ): & 2.5972 & ( & 78) \\
\hline (O8 & )$-(04$ & ): & 2.6285 & ( & 87) \\
\hline (08 & )$-(07$ & ): & 2.5920 & ( & 102) \\
\hline (O8 & )$-(08$ & ): & 2.5777 & ( & 107) \\
\hline (08 & )$-(09$ & ): & 2.5987 & ( & 78) \\
\hline (08 & )$-(010$ & ): & 2.6360 & ( & 84) \\
\hline (08 & )$-(010$ & ): & 2.6470 & ( & 91) \\
\hline (09 & )$-(04$ & ): & 2.6565 & ( & 54) \\
\hline (09 & )$-(04$ & ): & 2.6565 & ( & 54) \\
\hline (09 & )$-(07$ & ): & 2.5972 & ( & 78) \\
\hline (09 & )$-(07$ & ): & 2.5972 & ( & 78) \\
\hline (09 & )$-(08$ & ): & 2.5987 & ( & 78) \\
\hline (09 & )$-(08$ & ): & 2.5987 & ( & 78) \\
\hline (010 & )$-(03$ & ): & 2.6196 & ( & 78) \\
\hline (010 & )$-(05$ & ): & 2.5229 & ( & 69) \\
\hline (010 & )$-(06$ & ): & 2.5874 & ( & 87) \\
\hline (010 & )$-(08$ & ): & 2.6360 & ( & 84) \\
\hline (010 & )$-(08$ & ): & 2.6470 & ( & 91) \\
\hline (010 & )$-(010$ & ): & 2.5183 & ( & 62) \\
\hline
\end{tabular}




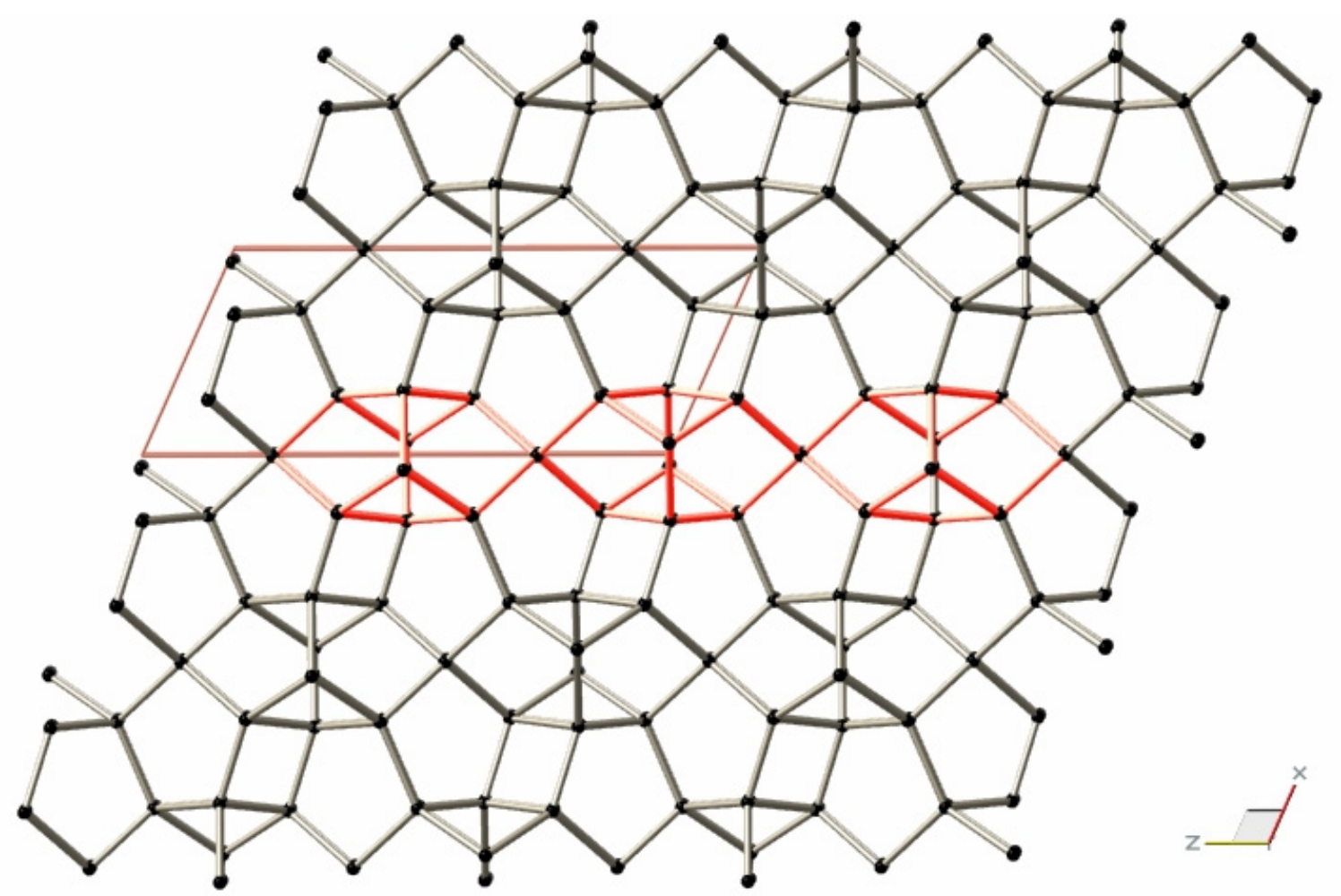

Fig.: Layer building unit of RUB-41. The basic building bolck of the layer is indicated in red. 


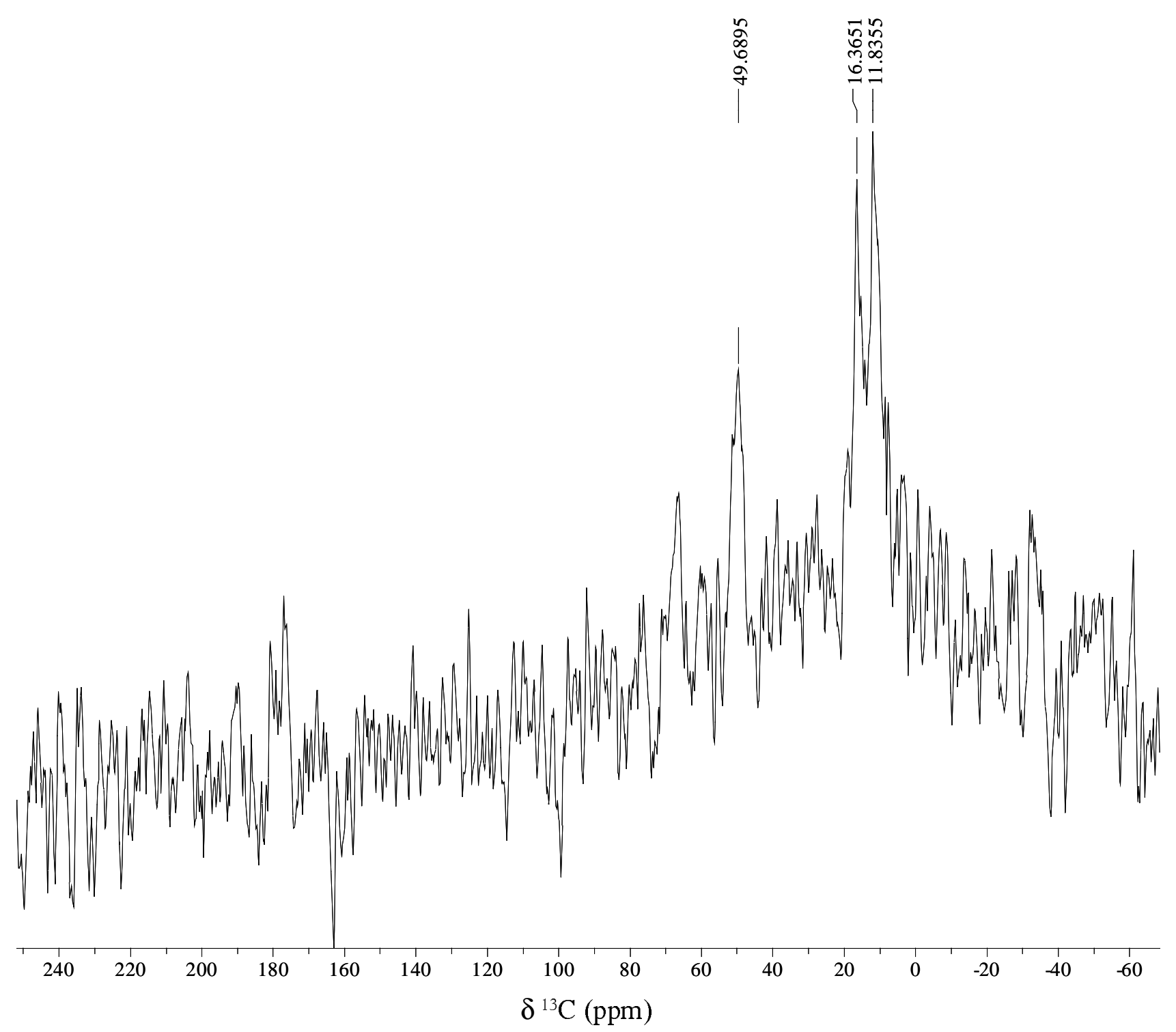

Fig.: ${ }^{13} C C P$ MAS NMR spectrum of the dimethydipropylammonium cation intercalated in RUB-39. 


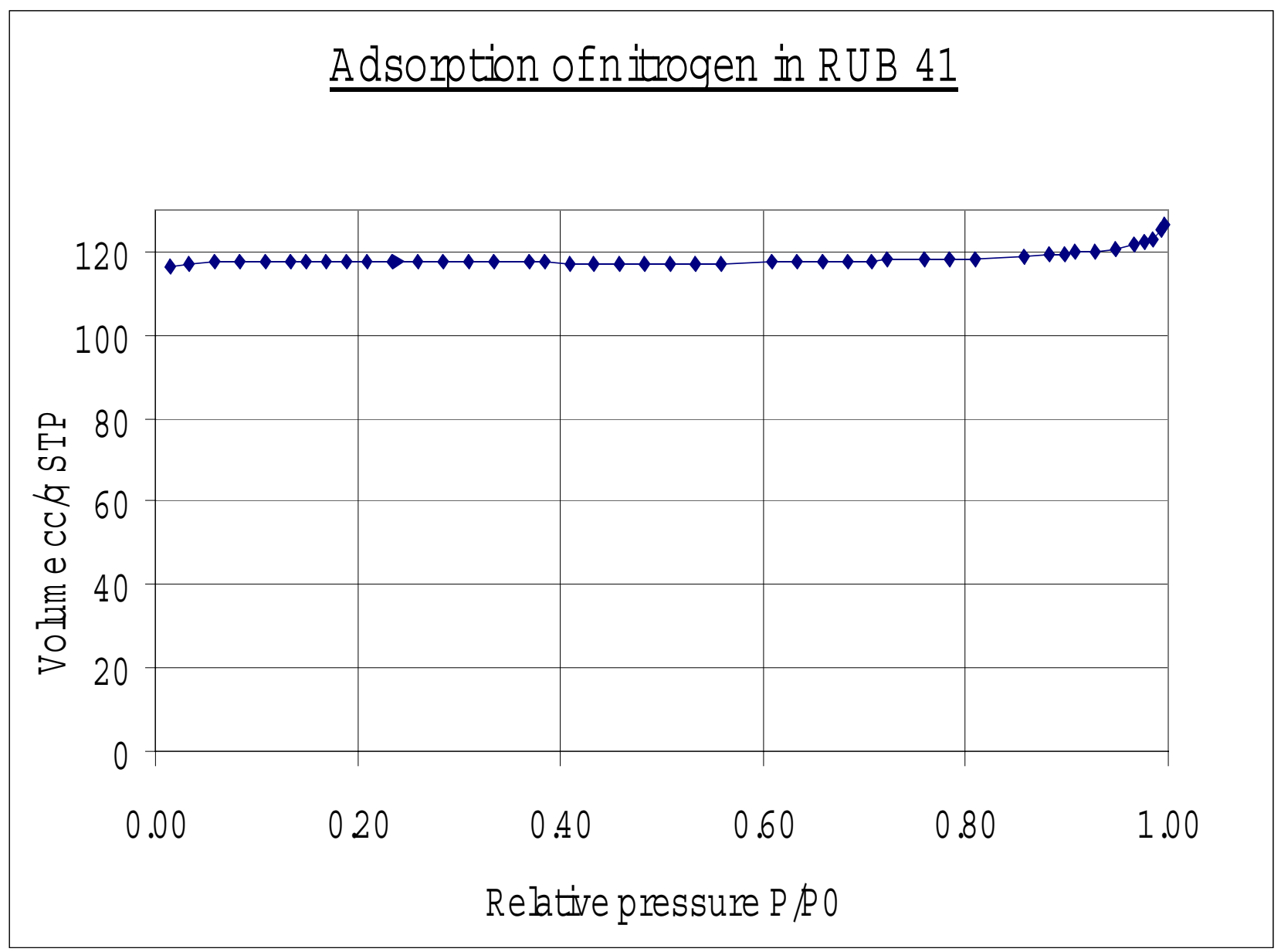

Fig.: Nitrogen adsorption isotherm for RUB-41. 
Adsorption of itbutane / $\mathrm{n}$ toutane on Rub-41

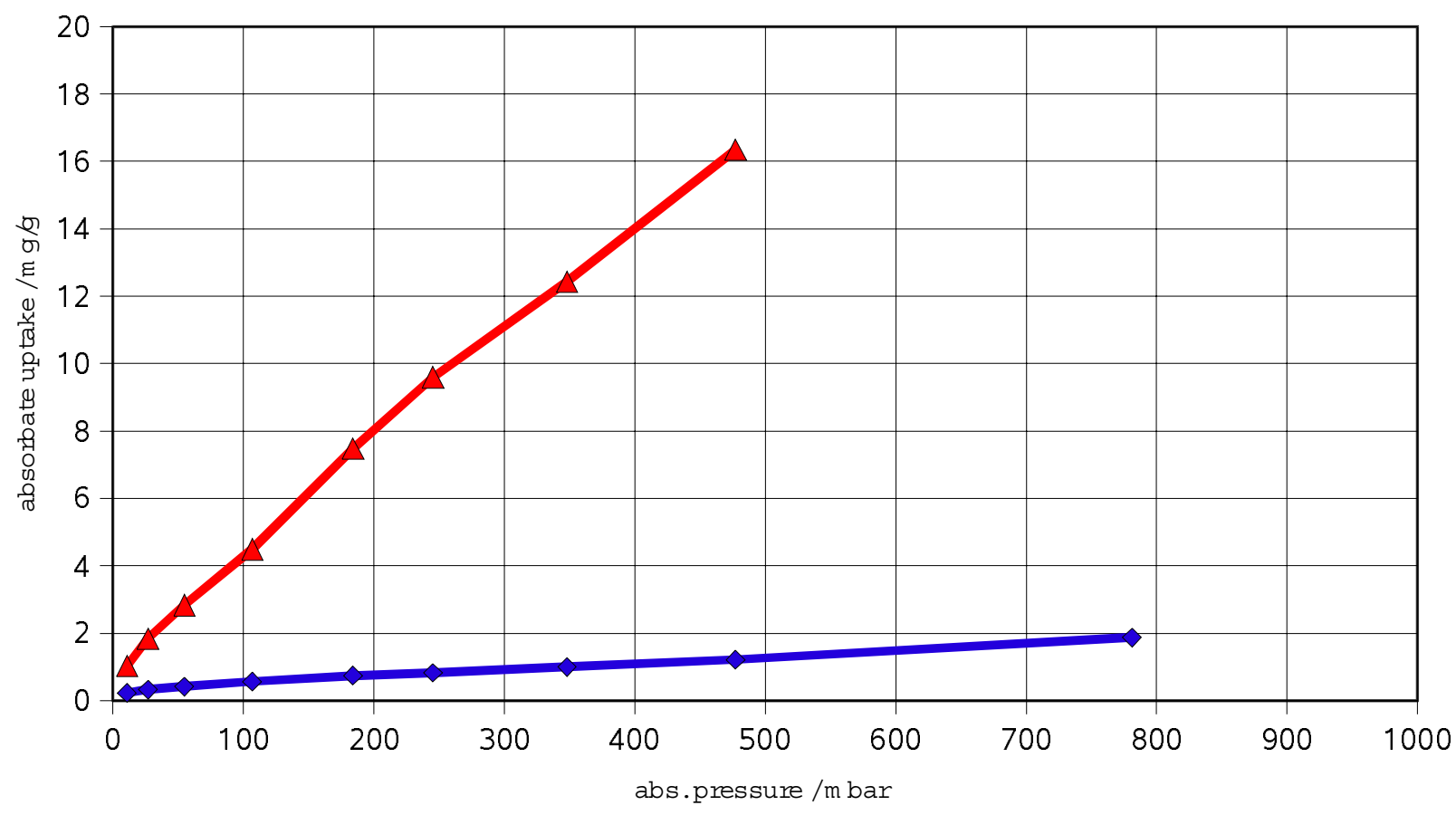

Fig.: Adsorption isotherm of n-butane (red) and i-butane (blue) taken at RT. 